\title{
A nomogram for determining the disease- specific survival in Ewing sarcoma: a population study
}

\author{
Jun Zhang ${ }^{1,2,3 \dagger}$, Zhenyu Pan ${ }^{1,2,4 \dagger}$, Jin Yang ${ }^{1,2+}$, Xiaoni Yan ${ }^{5}$, Yuanjie $\mathrm{Li}^{6}$ and Jun Lyu ${ }^{1,2^{*}}$ (D
}

\begin{abstract}
Background: We aimed to develop and validate a nomogram for predicting the disease-specific survival of Ewing sarcoma (ES) patients.

Methods: The Surveillance, Epidemiology, and End Results (SEER) program database was used to identify ES from 1990 to 2015, in which the data was extracted from 18 registries in the US. Multivariate analysis performed using Cox proportional hazards regression models was performed on the training set to identify independent prognostic factors and construct a nomogram for the prediction of the 3-, 5-, and 10-year survival rates of patients with ES. The predictive values were compared by using concordance indexes (C-indexes), calibration plots, integrated discrimination improvement (IDI), net reclassification improvement (NRI), and decision curve analysis (DCA).

Results: A total of 2,643 patients were identified. After multivariate Cox regression, a nomogram was established based on a new model containing the predictive variables of age, race, extent of disease, tumor size, and therapy of surgery. The new model provided better $\mathrm{C}$-indexes $(0.684$ and 0.704 in the training and validation cohorts, respectively) than the model without therapy of surgery $(0.661$ and 0.668 in the training and validation cohorts, respectively). The good discrimination and calibration of the nomogram were demonstrated for both the training and validation cohorts. NRI and IDI were also improved. Finally, DCA demonstrated that the nomogram was clinically useful.

Conclusion: We developed a reliable nomogram for determining the prognosis and treatment outcomes of patients with ES in the US. However, the proposed nomogram still requires external data verification in future applications, especially for regions outside the US.
\end{abstract}

Keywords: Nomogram, SEER, Disease-specific survival rate, Ewing sarcoma

\section{Background}

Ewing sarcoma (ES) is the second most common malignant primary osseous sarcoma in children and adolescents [1]. Bone ES constitutes a family of malignant small round blue cell tumors with neuroectodermal origins, among which $85-90 \%$ have the classic t $(11 ; 22)$ EWS/FLI1 translocation [1, 2]. The overall survival (OS) rate for ES has improved remarkably over the past two

\footnotetext{
* Correspondence: lujun2006@xjtu.edu.cn

†Jun Zhang, Zhenyu Pan and Jin Yang contributed equally to this work.

${ }^{1}$ Clinical Research Center, The First Affiliated Hospital of Xi'an Jiaotong University, Xi'an, Shaanxi, China

${ }^{2}$ School of Public Health, Xi'an Jiaotong University Health Science Center,

Xi'an, Shaanxi, China

Full list of author information is available at the end of the article
}

decades due to advances in multimodality therapies. In the US, the 5-year survival rates increased from $16 \%$ in the 1970 s to $39 \%$ in the 1990 s/early 2000 s among patients with metastatic disease. The survival parameter in patients with localized disease increased from 44 to $68 \%$ [3]. Despite these improvements, a large proportion of patients with ES still suffer from disease- or treatment-related morbidity or mortality. The early identification of high-risk patients can help provide adjuvant therapies or trial options. Given the clinical uniqueness of ES, prognostic tools are urgently needed to predict survival in ES patients accurately.

Nomograms are reliable and convenient tools for estimating tumor prognosis $[4,5]$. In this study, we 
aimed to establish a comprehensive prognostic evaluation system. The data of ES patients in the Surveillance, Epidemiology, and End Results (SEER) program database registries during 1990-2015 were screened and extracted. We then analyzed the extracted data and subsequently created and validated a nomogram containing significant and reliable variables for quantifying the survival of ES patients.

\section{Methods}

\section{Data source and inclusion criteria}

We queried the SEER program database for ES records from 1990 to 2015 that covers approximately $30 \%$ of the US population and includes cases from 18 populationbased registries [4]. Utilizing data from the SEER program does not require informed patient consent, and no case-identifying information is provided by the SEER cancer registries.

We searched for patients with ES by using the histological subtype code of "Ewing sarcoma" (9260/3) in the third edition of the International Classification of Diseases for Oncology. The patient demographic variables of interest included age at diagnosis (categorized into $\leq 30$ years old and $>30$ years old), sex, race, and marital status (categorized into married, single/domestic partner, or divorced/separated/widowed). A composite socioeconomic status (SES) score corresponding to the percentage of persons in the country living below the national poverty threshold in the official 2000 census [6] was divided into three levels by using previously reported cutoff points $[6,7]$, namely, $<10 \%$ (low poverty), $10-19.99 \%$ (moderate poverty), and $\geq 20 \%$ (high poverty). The year of diagnosis (YOD) was categorized into 1990s, 2000s and 2010s. EOD was categorized into confined, local invasion, metastasis, and unknown [8]. The primary site of ES was classified into extremity, axial skeleton, and others. Tumor size was grouped into $\leq 50 \mathrm{~mm}$ (small), $>50$ and $\leq 100 \mathrm{~mm}$ (intermediate), and $>100 \mathrm{~mm}$ (large) [9]. Surgery, radiotherapy, and chemotherapy were categorized into received and not received/unknown. Patients with missing or unknown of survival period were excluded.

\section{Statistical analysis and nomogram construction}

The categorical variables are expressed as frequencies and proportions and compared with the chi-square and Fisher's exact tests. Multivariate analysis was performed by using Cox proportional hazards regression models to determine the factors associated with survival. On the basis of the predictive model with identified prognostic factors, a nomogram was constructed for predicting the 3-, 5-, and 10-year survival rates of ES patients.

\section{Nomogram validation and performance evaluation}

The nomogram was validated by measuring the discrimination and calibration curves both internally (training cohort) and externally (validation cohort). Receiver operating characteristic (ROC) curves were generated to evaluate the performance of the nomogram on the basis of the areas under the ROC curves. The agreement between the predicted probability and actual outcome was evaluated via calibration plotting. The nomogram was subjected to bootstrapping validation $(1,000$ bootstrap resamples) to calculate a relatively corrected concordance index (C-index). The improvement in the predictive accuracy of the models with and without prognostic therapies was estimated by calculating the relative integrated discrimination improvement (IDI) and the net reclassification improvement (NRI), as described by Cook [10]. Finally, we evaluated the clinical usefulness and net benefit of the new predictive models by using decision curve analysis (DCA), as described by Vickers and Elkin [11].

Statistical analysis was conducted with SPSS (version 24.0; Chicago, IL, USA) and R (version 3.0.1; https:// www.r-project.org/) softwares. $P$ values $<0.05$ of the two-sided tests were considered statistically significant.

\section{Results}

\section{Demographic baseline characteristics}

The application of the inclusion and exclusion criteria listed in the Materials and Methods resulted in the identification of 2,643 patients with ES in the SEER program database. The survival period was known for all of the included patients. For nomogram construction and validation, we randomly assigned 70 and $30 \%$ of the patients to the training $(n=1,850)$ and validation $(n=793)$ cohorts, respectively. The majority of patients were $\leq 30$ years old (78.4 and $79.8 \%$ in the training and validation cohorts, respectively) and male (58.9 and 62.7\%), white (88.1 and $88.8 \%$ ), had a marital status of single/domestic partner (76.3 and 79.8\%), had been diagnosed in the $2000 \mathrm{~s}$ or 2010s (82.4 and 82.1\%), had unknown EOD (67.1 and $67.0 \%)$, and had undergone surgery (60.9 and 59.9\%) and chemotherapy (91.0 and 93.9\%). The clinicopathological characteristics of all patients are listed in Table 1.

\section{Multivariate cox regression analysis results}

Multivariate models were developed to identify independent prognostic variables. Sex, marital status, SES score, YOD, primary site, radiotherapy, and chemotherapy were not associated with the significant differences in survival. Thus, age at diagnosis, race, EOD, tumor size, and surgery were subjected to multivariate Cox regression analysis. The multivariate analysis demonstrated that age at diagnosis $>30$ years old (adjusted hazard ratio [12], 2.153; 95\% confidence interval [CI], 1.812 to 2.558 ; 
Table 1 Socio-demographic and clinical characteristics of patients in the study

\begin{tabular}{lll}
\hline Variable $\mathrm{n}(\%)$ & $\begin{array}{ll}\text { Training Cohort } \\
(n=1850)\end{array}$ & $\begin{array}{l}\text { Validation Cohort } \\
(n=793)\end{array}$ \\
\hline
\end{tabular}

\begin{tabular}{lll}
\hline Age & & \\
$\leq 30$ years & $1451(78.4)$ & $633(79.8)$ \\
$>30$ years & $399(21.6)$ & $160(20.2)$ \\
Sex & & \\
Male & $1089(58.9)$ & $497(62.7)$ \\
Female & $761(41.1)$ & $296(37.3)$ \\
Race & & \\
White & $1630(88.1)$ & $704(88.8)$ \\
Black & $65(3.5)$ & $34(4.3)$ \\
Other & $155(8.4)$ & $55(6.9)$
\end{tabular}

Marital status

Married
Single/Domestic Partner
DSW
SES

Low poverty
Medium poverty
High poverty
YOD

1990s
2000s and 2010s
EOD

Confined
Local Invasion
Metastasis
Unknown
Site
Extremity

Axial Skeleton

\section{Tumor Size}

$\leq 50 \mathrm{~mm}$
$>50 \mathrm{~mm}-100 \mathrm{~mm}$
$>100 \mathrm{~mm}$
Surgery
Yes

\section{NO/Unknown}

Radiotherapy

Yes

No

$\begin{array}{ll}341(18.4) & 131(16.5) \\ 1412(76.3) & 633(79.8) \\ 97(5.2) & 29(3.7)\end{array}$

$\begin{array}{ll}744(40.2) & 315(39.7) \\ 986(53.3) & 425(53.6) \\ 120(6.5) & 53(6.7)\end{array}$

$\begin{array}{ll}325(17.6) & 142(17.9) \\ 1525(82.4) & 651(82.1) \\ 56(3.0) & 27(3.4) \\ 374(20.2) & 147(18.5) \\ 179(9.7) & 88(11.1) \\ 1241(67.1) & 531(67.0) \\ & \\ 420(22.7) & 186(23.5) \\ 718(38.8) & 295(37.2) \\ 712(38.5) & 312(39.3)\end{array}$

$302(16.3) \quad 138(17.4)$

529(28.6) $\quad 216(27.2)$

$1019(55.1) \quad 439(55.4)$

$1126(60.9)$

$475(59.9)$

$724(39.1) \quad 318(40.1)$

$871(47.1) \quad 353(44.5)$

$979(52.9) \quad 440(55.5)$

Chemotherapy
Table 1 Socio-demographic and clinical characteristics of patients in the study (Continued)

\begin{tabular}{lll}
\hline Variable $\mathrm{n}(\%)$ & $\begin{array}{l}\text { Training Cohort } \\
(n=1850)\end{array}$ & $\begin{array}{l}\text { Validation Cohort } \\
(n=793)\end{array}$ \\
\hline NO/Unknown & $167(9.0)$ & $48(6.1)$ \\
\hline
\end{tabular}

Abbreviations: DSW Divorced, separated and widowed, YOD Year of diagnosis, EOD Extend of disease, SES Socioeconomic Status

$p<0.001$ ), being black (aHR, 1.497; 95\% CI, 1.054 to 2.128; $p<0.05$ ), metastasis (aHR, 4.839; 95\% CI, 2.780 to 8.424; $p<0.001$ ), unknown EOD (aHR, 2.127; 95\% CI, 1.246 to $3.632 ; p<0.01$ ), tumor size $>50$ and $\leq 100 \mathrm{~mm}$ (aHR, 1.469; 95\% CI, 1.105 to $1.953 ; p<0.01$ ), tumor size $>100 \mathrm{~mm}$ (aHR, 2.273; 95\% CI, 1.755 to $2.945 ; p<0.001$ ), and nonsurgical treatment (aHR, 1.951; 95\% CI, 1.670 to $2.280 ; p<0.001)$ were independent negative predictors of disease-specific survival (DSS). The multivariate analyses of DSS in the training set are listed in Table 2.

\section{Nomogram construction}

The results of the logistic regression model listed in Table 2 were utilized to construct a nomogram (Fig. 1). Each predictor was included in its line according to that scale. The total points on the nomogram were added

Table 2 Multivariate analyses of disease-specific survival in the training set

\begin{tabular}{|c|c|c|c|}
\hline \multirow[t]{2}{*}{ Variable } & \multicolumn{3}{|c|}{ Multivariate analysis } \\
\hline & $\mathrm{aHR}$ & $95 \% \mathrm{Cl}$ & $p$-value \\
\hline \multicolumn{4}{|l|}{ Age } \\
\hline$\leq 30$ years & Reference & & \\
\hline$>30$ years & 2.153 & $1.812-2.558$ & $0.000^{* * *}$ \\
\hline \multicolumn{4}{|l|}{ Race } \\
\hline White & Reference & & \\
\hline Black & 1.497 & $1.054-2.128$ & $0.024^{*}$ \\
\hline Other & 1.177 & $0.894-1.548$ & 0.245 \\
\hline \multicolumn{4}{|l|}{ EOD } \\
\hline Confined & Reference & & \\
\hline Local Invasion & 1.692 & $0.977-2.932$ & 0.061 \\
\hline Metastasis & 4.839 & $2.780-8.424$ & $0.000^{* * *}$ \\
\hline Unknown & 2.127 & $1.246-3.632$ & $0.006^{* *}$ \\
\hline \multicolumn{4}{|l|}{ Tumor Size } \\
\hline$\leq 50 \mathrm{~mm}$ & Reference & & \\
\hline$>50 \mathrm{~mm}-100 \mathrm{~mm}$ & 1.469 & $1.105-1.953$ & $0.008^{* *}$ \\
\hline$>100 \mathrm{~mm}$ & 2.273 & $1.755-2.945$ & $0.000^{* * *}$ \\
\hline \multicolumn{4}{|l|}{ Surgery } \\
\hline Yes & Reference & & \\
\hline NO/Unknown & 1.951 & $1.670-2.280$ & $0.000^{* * *}$ \\
\hline
\end{tabular}

Abbreviations: SEER Surveillance, Epidemiology, and End Result, aHR Adjusted hazard ratio, EOD Extend of disease Note: ${ }^{*} p<0.05,{ }^{* *} p<0.01,{ }^{* * *} p<0.001$ 


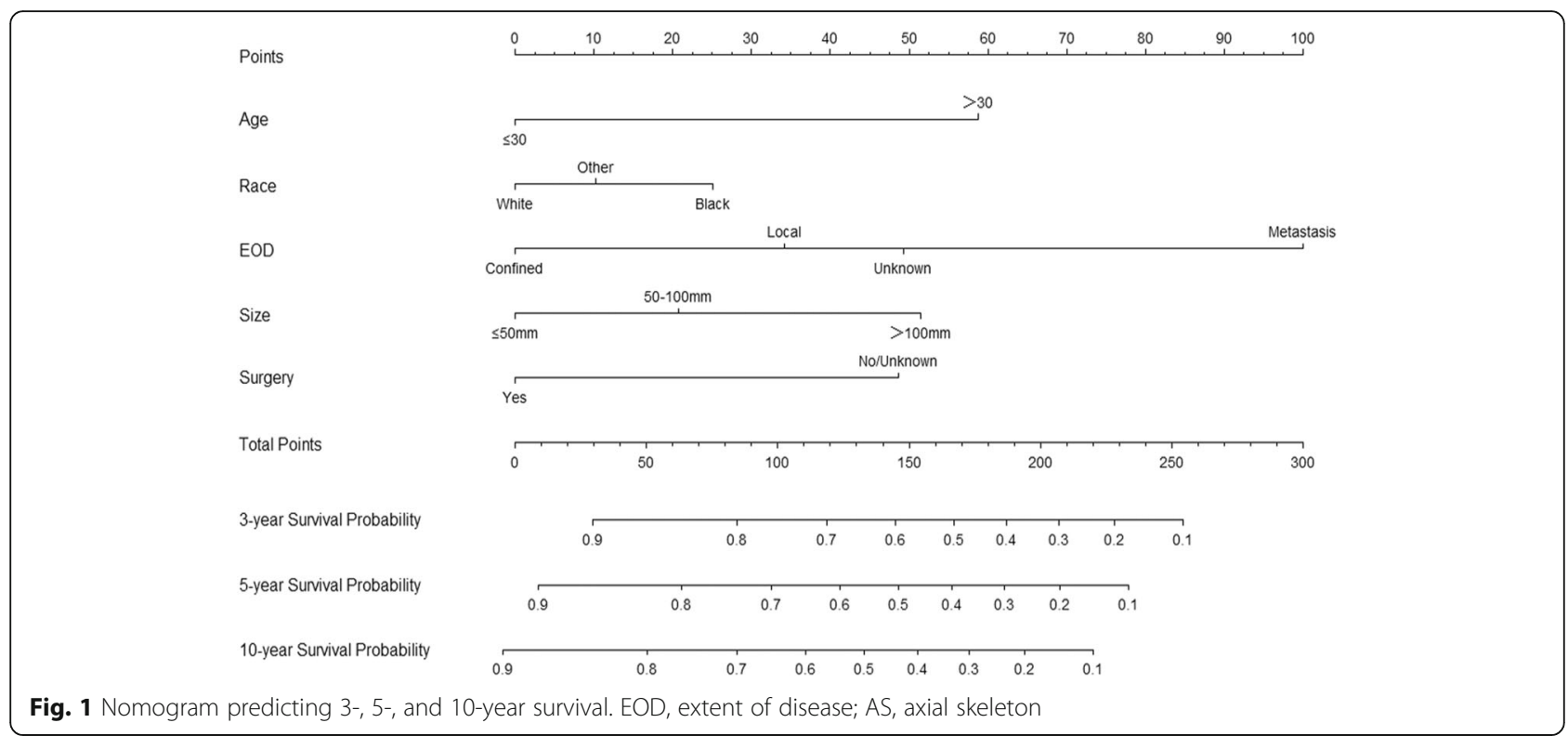

and then converted into the probability of 3-, 5-, and 10year survival with guidance from the linear parallel lines. The nomogram showed that metastasis, which had the largest absolute values, was the strongest contributor to the risk of prognosis, followed by age $>30$ years old, large tumor $(>100 \mathrm{~mm})$, nonsurgical treatment, local and unknown EOD, being black, intermediate tumor size (>50 and $\leq 100 \mathrm{~mm}$ ), and being male. Meanwhile, the protective factors included age $\leq 30$ years old, being white, confined tumor, small size tumor ( $\leq 50 \mathrm{~mm})$, and surgery performed.

\section{Performance of the nomogram}

Based on the C-index analysis of the SEER training cohort, the nomogram provided relatively high $\mathrm{C}$-indexes for the 3-, 5-, and 10-year survivals at 0.721, 0.713, and 0.699, respectively; the corresponding values for the external validation cohort were also high at $0.721,0.718$, and 0.723 . These findings indicated that the model had good discriminative ability (Fig. 2).

\section{Validation of the nomogram}

The new model for the established nomogram included the following variables that were entered into the multivariate Cox regression analysis: age at diagnosis, race, EOD, tumor size, and surgery. The new model that included therapy of surgery provided better C-indexes ( 0.684 and 0.704 in the training and validation cohorts, respectively) than that of the model without surgery

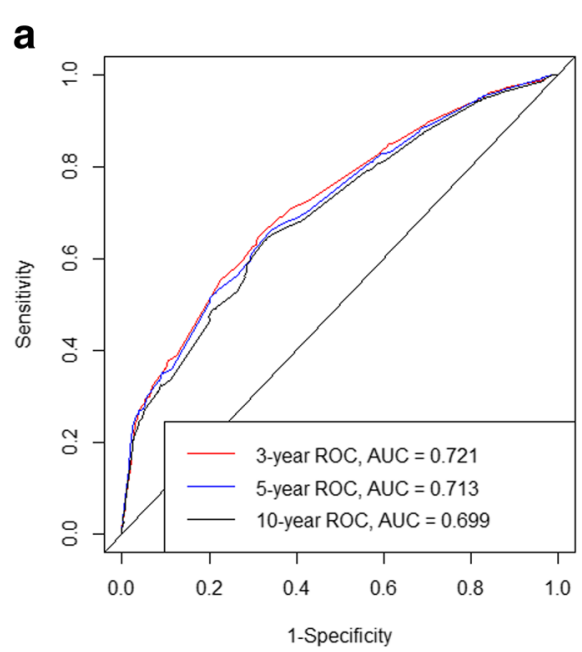

b

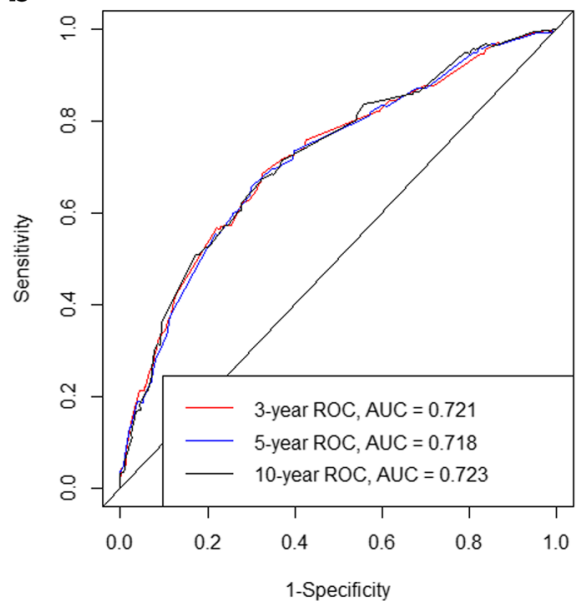

Fig. 2 ROC curves. ROC curve analyses were generated to test the performance evaluating of the newly established nomogram, by the areas under the ROC curves (AUC). $\mathbf{a}$ came from the training set, and $\mathbf{b}$ came from the validation set 
(0.661 and 0.668). A high C-index indicates good ability to separate patients with different survival outcomes. The calibration curves in Fig. 3 depict the calibration of the new model in terms of the agreement between the predicted probabilities and observed outcomes for 3-, 5-, and 10-year survival.

The NRI values were 0.361 ( $95 \% \mathrm{CI}, 0.241$ to 0.525 ), 0.481 ( $95 \%$ CI, 0.260 to 0.562 ), and 0.520 (95\% CI, 0.350 to 0.569 ) for 3,5 , and 10 years of follow-up examinations in the training cohort, respectively. In the validation cohort, the NRI values for 3,5 , and 10 years of follow-up were 0.351 ( $95 \% \mathrm{CI}, 0.242$ to 0.502$), 0.458$ (95\% CI, 0.320 to 0.541 ), and 0.494 (95\% CI, 0.350 to 0.670 ), respectively. These results showed that the new model exhibited superior predictive performance compared with the model without the therapy of surgery. Similarly, the IDI values for 3,5 , and 10 years of follow-up examinations were $0.026,0.028$, and 0.029 in the training cohort and 0.021 , 0.023 , and 0.025 in the validation cohort, respectively.

\section{Clinical use}

DCA graphically showed the large net benefits of the new model for predicting 3-, 5-, and 10-year survival (Fig. 4) to verify its clinical utilization and impact in practical decision-making.

\section{Discussion}

ES is an rare and aggressive type of malignancy that normally develops in young patients from childhood to early adulthood [13]. ES is the second most common primary malignant bone tumor in people younger than 30 years (second only to osteosarcoma) and the most common primary malignant bone tumor in those younger than 10 years. The annual incidence of ES among Caucasians is less than 3 per 1,000,000 [3], thereby indicating that data from single-center studies cannot provide adequate sample sizes. Therefore, this study was based on a largesample database of the SEER program, which initially started with eight registries in 1973 and has continuously added other participating sites over time. At present, the database includes 18 geographically diverse areas representing $26 \%$ of the US population with efforts to reflect the racial, economic, and social diversity of the country as a whole $[2,6,14]$. The neoadjuvant chemoradiation treatment of ES began in the early 1990s [15]. To obtain reliable research results, we identified 2,643 patients with ES in the SEER program database from 1990 to 2015. ES mostly occurs in young people. In our study, most of the patients were $\leq 30$ years old, accounting for 78.4 and $79.8 \%$ in the training and validation cohorts, respectively. Table 1 presents that most of the patients were male, white, had a marital status of single/ domestic partner, diagnosed in the 2000s or 2010s, treated with surgery, and treated with chemotherapy; these results were consistent with previous research findings [16-19]. Although ES has the highest incidence in people under the age of 30 [20], the prognosis is better for those with a younger age of onset and worse for those with a higher age of onset [1]. Similarly, in our nomogram (Fig. 1), the prognosis of people older than 30 was worse than that of people younger than 30 . Regarding the cause of this phenomenon, Lee et al. [19] and Grevener et al. [21] found that adult patients received few cases of chemotherapy, and older patients were more likely to have multiple comorbidities, including diabetes, high blood pressure, and secondary cancer, which complicated the situation.

The long-term survival rate of ES for nonmetastatic disease at presentation has improved from 10 to $15 \%$ to $60-70 \%$ since the early 1990 s through the application of multimodality approaches, including surgery, radiotherapy, and neoadjuvant chemotherapy [12, 22, 23]. However, ES exhibits an aggressive behavior that often results in lung metastasis, which is a poor prognostic factor given that only $20 \%$ of patients with metastases can survive for a long time $[1,2,20]$. The early identification of high-risk ES patients is helpful in providing adjuvant treatment or trials. Existing clinical staging systems only consider tumor size and histological metastasis. For example, the staging system of the American Joint Committee on Cancer can only estimate the limited clinical risk of ES. Therefore, the use of Cox regression analysis and the developed nomogram provides a comprehensive predictive model that includes not only the system demographics but also the therapy of surgery and other clinical parameters.

A nomogram is a convenient graphical representation of a mathematical model. It provides an intuitive way to combine important factors and predict a specific endpoint. The nomogram is also a reliable tool for quantifying risk and widely used in applied tumor prognoses. A well-developed clinical nomogram is a popular decisionmaking tool that can be used to predict the outcome of an individual and benefit both clinicians and patients [24]. Nomograms in many studies $[2,16,17,20,25]$ indicate that being black and aged appear to be high-risk factors. However, small size tumor and surgery treatment demonstrate improved outcomes in DSS for ES. This trend is understandable given the aggressive therapies needed to treat such disease. Patients with metastatic diseases at the initial presentation have worse prognoses than those with confined diseases $[2,13,18,25]$. Knowledge of these features will be helpful in clinical decisions.

Similar to previous studies [10, 26, 27], we applied IDI and NRI to evaluate whether the newly constructed prognostic model performed well and whether it should be used in clinical practice. Compared with radiotherapy and chemotherapy, surgery is 
a

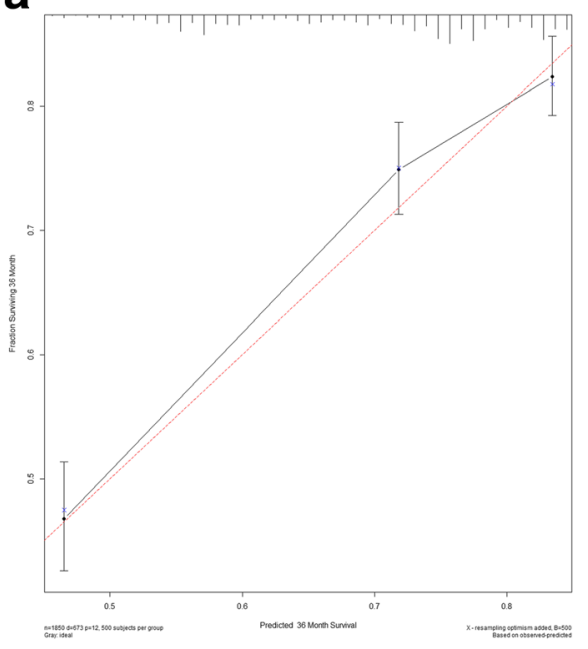

C

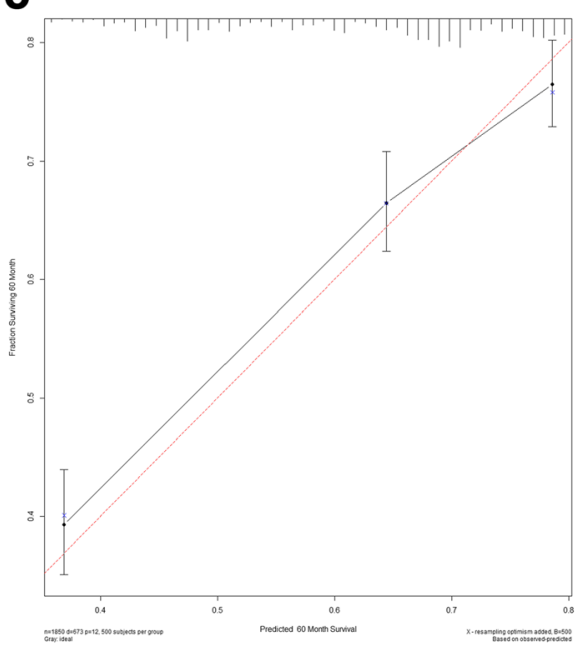

e

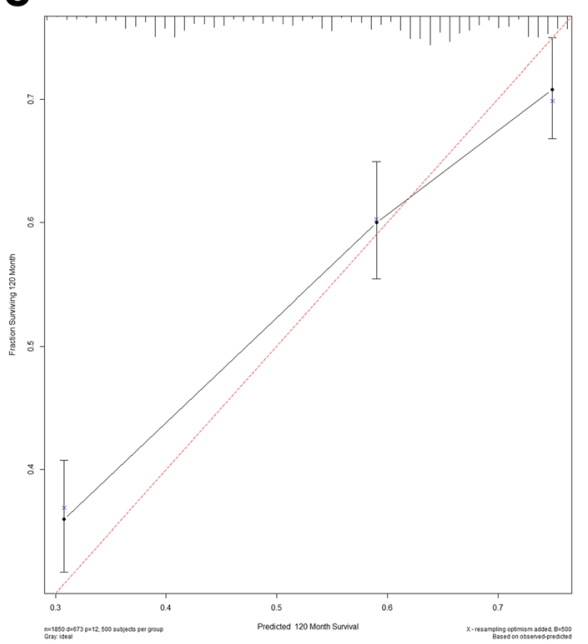

b

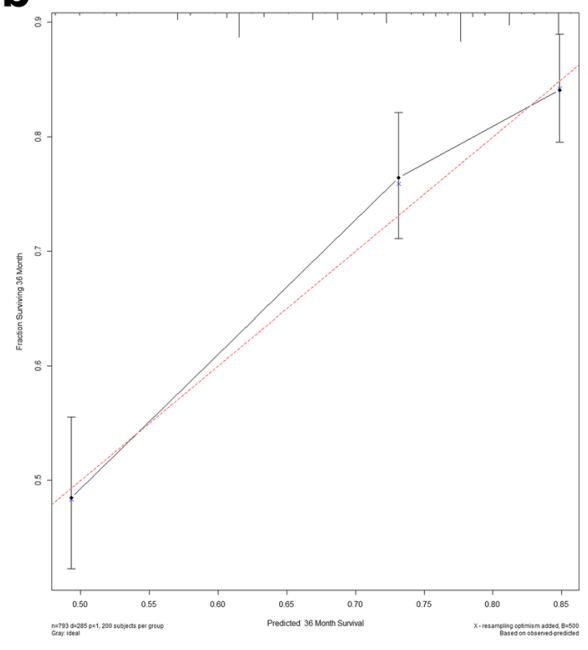

d

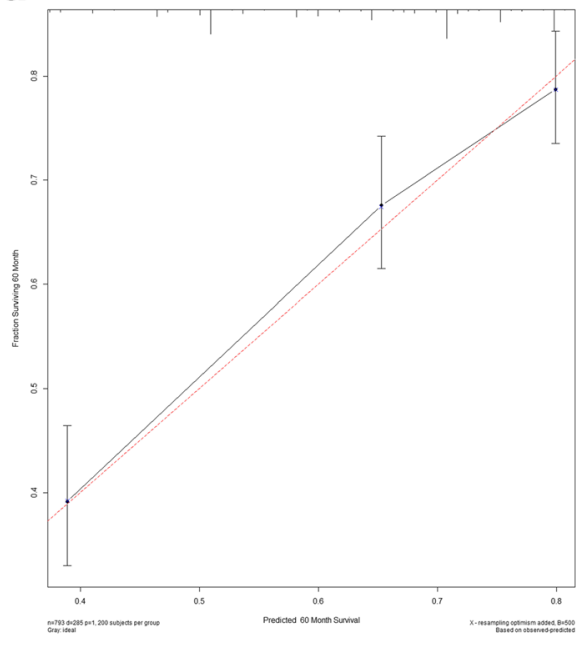

f

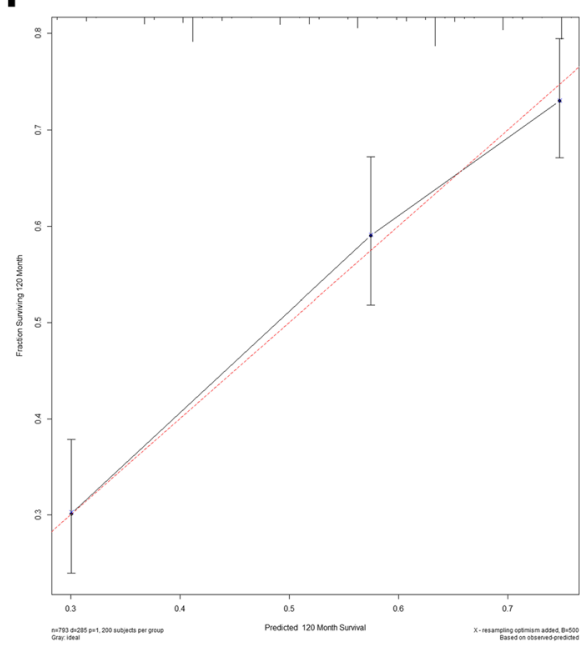

Fig. 3 Calibration curves for 3-, 5-, and 10-year survival. Calibration curves depict the calibration of each model in terms of the agreement between the predicted probabilities and observed outcomes of the training set $(\mathbf{a}, \mathbf{c}, \mathbf{e})$ and validation set (b, $\mathbf{d}$, $\mathbf{f}$ ) 

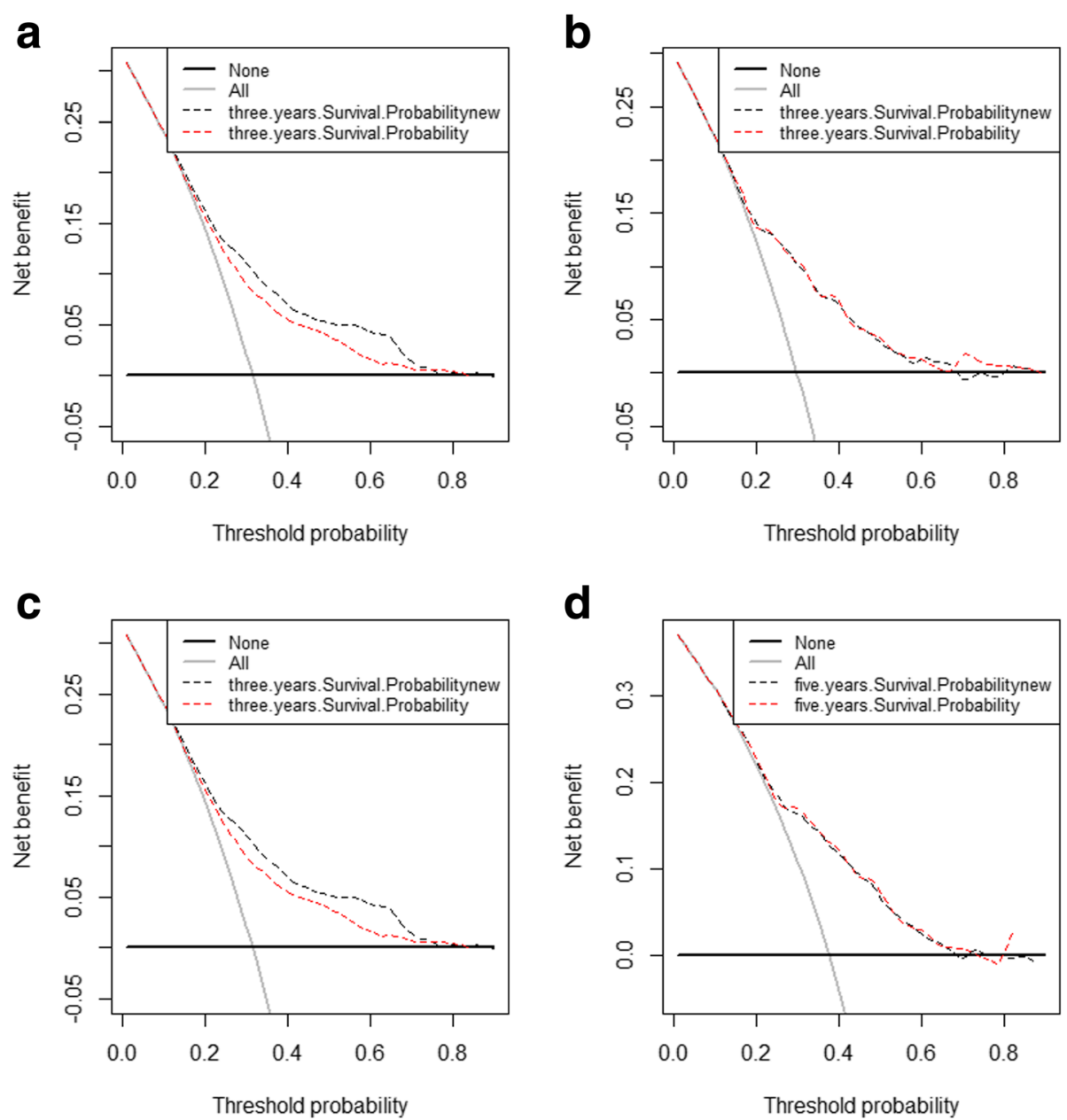

e

$\mathbf{f}$
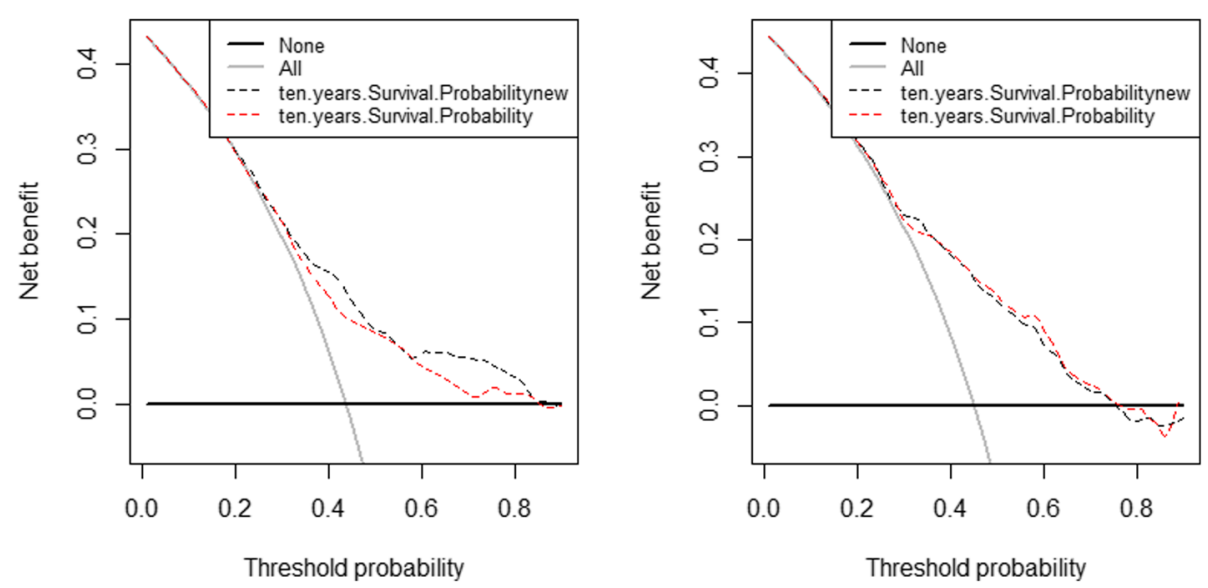

Fig. 4 Decision curve analysis of the training set $(\mathbf{a}, \mathbf{c}, \mathbf{e})$ and validation set $(\mathbf{b}, \mathbf{d}, \mathbf{f})$ for 3-, 5-, and 10-year survival. In the figure, the blue dotted line represents the DCA of new model, contrastively, the red dotted line represents the DCA of model without therapies. All = Assume all ES patients survive, None $=$ Assume none ES patient survives

the most effective means of treating ES [2, 18]. The new model containing the therapy of surgery showed good discrimination and calibration, in which both IDI and NRI for 3, 5, and 10 years of follow-up examinations showed improved C-index, as mentioned in the Results section. 
Finally, our newly constructed nomogram model included a wide range of clinical risk factors, namely, age at diagnosis, race, EOD, tumor size, and surgery, which were easily available and routinely collected from historical records. Figure 4 shows the results of our DCA, wherein the abscissa and ordinate are the threshold probability and net benefit rate, respectively [28-31]. To the best of our knowledge, this study is the first to use IDI, NRI, and DCA in the verification of the predictive abilities of nomograms for ES. Thus, the nomogram is helpful to accurately predict the 3-, 5-, and 10-year survivals of ES patients.

\section{Limitations}

First, important prognostic factors, such as tumor markers and the expression of the TP53 gene, were not available in the SEER database. Second, information was not available for some of the cases. Hence, we could only define the subclassifications as unknown, such as for EOD and tumor size. Third, similar to other malignant bone tumors, ES showed unavailable AJCC/TNM data in the SEER database that might have affected the diagnostic and predictive accuracy of our new tool [32]. Finally, rather than representing absolutely accurate prognoses, the predicted values calculated from the nomogram were only suitable for interpretation by clinicians. Future studies can use the present findings to develop a well-accepted risk prediction tool for ES [33].

\section{Conclusions}

Nomograms are an important component of modern medical decision-making. We developed a reliable nomogram for determining the prognosis and treatment outcomes of ES patients in the US. However, external data verification is still required in future applications, especially for regions outside the US.

\section{Abbreviations}

AUC: Area under the curve; DCA: Decision curve analysis; DSW: Divorced, separated and widowed; ES: Ewing sarcoma; IDI: Integrated discrimination improvement; NRI: Net reclassification improvement; OS: Overall survival; SEER: Surveillance, Epidemiology, and End Results; SES: Socioeconomic status; YOD: Year of diagnosis

\section{Acknowledgements}

The authors acknowledge the efforts of the SEER program in the creation of the SEER database.

\section{Authors' contributions \\ $J L$ and $J Z$ conceived the study idea. ZYP, JY and XNY performed data mining, and statistical analyses. YJL and ZYP interpreted results of statistical analyses. JZ drafted the initial manuscript. JL made critical comment and revision for the initial manuscript. JL had primary responsibility for the final content. JZ, YZP and JY are co-first authors. JL is correspondence author. All authors reviewed and approved the final manuscript.}

\section{Funding}

This study was funded by National Social Science Foundation of China (No.16BGL183), and the Research Fund of Health Bureau of Xi'an (No.QFO1330). None of the funding sources were involved in design of the study, data collection and analysis, interpretation of results, writing of the manuscript, or in the decision to submit the manuscript for publication.

\section{Availability of data and materials}

Limited Use Agreement for Surveillance, Epidemiology, and End Results (SEER) Program (https://seer.cancer.gov) SEER*Stat Database: released in April 2017 based on the November 2016 submission. The data can be used publicly.

\section{Ethics approval and consent to participate}

The SEER program database is publicly available and provides de-identified case data. Owing to the data of SEER program is anonymous and cancer is a reportable disease in every state, the requirement for informed consent was therefore waived by the $s$ in the US [34].

\section{Consent for publication}

Not Applicable.

\section{Competing interests}

The authors declare that they have no competing interests.

\section{Author details}

${ }^{1}$ Clinical Research Center, The First Affiliated Hospital of Xi'an Jiaotong University, Xi'an, Shaanxi, China. ${ }^{2}$ School of Public Health, Xi'an Jiaotong University Health Science Center, Xi'an, Shaanxi, China. ${ }^{3}$ Department of Orthopaedics, Baoji Municipal Central Hospital, Baoji, Shaanxi, China. ${ }^{4}$ Department of Pharmacy, The Affiliated Children Hospital of Xi'an Jiaotong University, Xi'an, Shaanxi, China. ${ }^{5}$ Department of Gastroenterology, The First Affiliated Hospital of Xi'an Jiaotong University, Xi'an, Shaanxi, China.

${ }^{6}$ Department of Human Anatomy, Histology and Embryology, School of Basic Medical Sciences, Xi'an Jiaotosng University Health Science Center, Xi'an, Shaanxi, China.

Received: 4 December 2018 Accepted: 30 June 2019

Published online: 05 July 2019

\section{References}

1. Rodriguez-Galindo C, Navid F, Liu T, Billups CA, Rao BN, Krasin MJ. Prognostic factors for local and distant control in Ewing sarcoma family of tumors. Ann Oncol. 2008;19(4):814-20.

2. Arshi A, Sharim J, Park DY, Park HY, Yazdanshenas H, Bernthal NM, Shamie AN. Prognostic determinants and treatment outcomes analysis of osteosarcoma and Ewing sarcoma of the spine. Spine J. 2017;17(5):645-55

3. Esiashvili N, Goodman M, Marcus RB Jr. Changes in incidence and survival of Ewing sarcoma patients over the past 3 decades: surveillance epidemiology and end results data. J Pediatr Hematol Oncol. 2008;30(6): 425-30.

4. Lin Z, Yan S, Zhang J, Pan Q. A nomogram for distinction and potential prediction of liver metastasis in breast Cancer patients. J Cancer. 2018;9(12): 2098-106.

5. Balachandran VP, Gonen M, Smith JJ, DeMatteo RP. Nomograms in oncology: more than meets the eye. Lancet Oncol. 2015;16(4):e173-80.

6. Wu J, Sun H, Li J, Guo Y, Zhang K, Lang C, Zou C, Ma H. Increased survival of patients aged 0-29 years with osteosarcoma: a period analysis, 1984-2013. Cancer Med. 2018;7(8):3652-61.

7. Ma H, Sun H, Sun X. Survival improvement by decade of patients aged 0-14 years with acute lymphoblastic leukemia: a SEER analysis. Sci Rep. 2014;4:4227.

8. Wang Z, Li S, Li Y, Lin N, Huang X, Liu M, Pan W, Yan X, Sun L, Li H, et al. Prognostic factors for survival among patients with primary bone sarcomas of small bones. Cancer Manag Res. 2018;10:1191-9.

9. Duchman KR, Gao Y, Miller BJ. Prognostic factors for survival in patients with Ewing's sarcoma using the surveillance, epidemiology, and end results (SEER) program database. Cancer Epidemiol. 2015;39(2):189-95.

10. Cook NR. Comments on 'Evaluating the added predictive ability of a new marker: from area under the ROC curve to reclassification and beyond' by M. J. Pencina et al., statistics in medicine (DOI: 10.1002/sim.2929). Stat Med. 2008;27(2):191-5.

11. Vickers AJ, Elkin EB. Decision curve analysis: a novel method for evaluating prediction models. Med Decis Mak. 2006;26(6):565-74.

12. Paulussen M, Ahrens S, Dunst J, Winkelmann W, Exner GU, Kotz R, Amann G, Dockhorn-Dworniczak B, Harms D, Muller-Weihrich S, et al. Localized Ewing 
tumor of bone: final results of the cooperative Ewing's sarcoma study CESS 86. J Clin Oncol. 2001;19(6):1818-29.

13. Krakorova DA, Kubackova K, Dusek L, Tomas T, Janicek P, Tucek S, Prausova J, Kiss I, Zambo I. Advantages in prognosis of adult patients with Ewing sarcoma: 11-years experiences and current treatment management. Pathol Oncol Res. 2018:24(3):623-30.

14. Mirabello L, Troisi RJ, Savage SA. Osteosarcoma incidence and survival rates from 1973 to 2004: data from the surveillance, epidemiology, and end results program. Cancer. 2009;115(7):1531-43.

15. Gaspar N, Hawkins DS, Dirksen U, Lewis IJ, Ferrari S, Le Deley MC, Kovar H, Grimer R, Whelan J, Claude L, et al. Ewing sarcoma: current management and future approaches through collaboration. J Clin Oncol. 2015;33(27):3036-46.

16. Cheung MR. Optimization of predictors of Ewing sarcoma cause-specific survival: a population study. Asian Pac J Cancer Prev. 2014;15(10):4143-5.

17. Campbell K, Shulman D, Janeway KA, DuBois SG. Comparison of epidemiology, clinical features, and outcomes of patients with reported Ewing sarcoma and PNET over 40 years justifies current WHO classification and treatment approaches. Sarcoma. 2018;2018:1712964.

18. Wan $Z H$, Huang $Z H$, Chen LB. Survival outcome among patients with Ewing's sarcoma of bones and joints: a population-based cohort study. Sao Paulo Med J. 2018;136(2):116-22.

19. Lee J, Hoang BH, Ziogas A, Zell JA. Analysis of prognostic factors in Ewing sarcoma using a population-based cancer registry. Cancer. 2010;116(8):1964-73.

20. Chakraborty D, Rangamani S, Kulothungan V, Chaturvedi M, Stephen S, Das P, Sudarshan KL, Janani Surya R, Sathish Kumar K, John A, et al. Trends in incidence of Ewing sarcoma of bone in India - evidence from the National Cancer Registry Programme (1982-2011). J Bone Oncol. 2018;12:49-53.

21. Grevener K, Haveman LM, Ranft A, van den Berg H, Jung S, Ladenstein R, KlcoBrosius S, Juergens $\mathrm{H}$, Merks JH, Dirksen U. Management and outcome of Ewing sarcoma of the head and neck. Pediatr Blood Cancer. 2016;63(4):604-10.

22. Ataergin S, Ozet A, Solchaga L, Turan M, Beyzadeoglu M, Oysul K, Arpaci F, Komurcu S, Surenkok S, Ozturk M. Long-lasting multiagent chemotherapy in adult high-risk Ewing's sarcoma of bone. Med Oncol. 2009;26(3):276-86.

23. Akagunduz OO, Kamer SA, Kececi B, Demirag B, Oniz H, Kantar M, Cetingul $\mathrm{N}$, Sabah $\mathrm{D}$, Anacak $\mathrm{Y}$. The role of radiotherapy in local control of nonextremity Ewing sarcomas. Tumori. 2016;102(2):162-7.

24. Liu RZ, Zhao ZR, Ng CS. Statistical modelling for thoracic surgery using a nomogram based on logistic regression. J Thorac Dis. 2016;8(8):E731-6.

25. Fukushima T, Ogura K, Akiyama T, Takeshita K, Kawai A. Descriptive epidemiology and outcomes of bone sarcomas in adolescent and young adult patients in Japan. BMC Musculoskelet Disord. 2018;19(1):297.

26. Chen LD, Liang JY, Wu H, Wang Z, Li SR, Li W, Zhang XH, Chen JH, Ye JN, Li $X$, et al. Multiparametric radiomics improve prediction of lymph node metastasis of rectal cancer compared with conventional radiomics. Life Sci. 2018:208:55-63

27. Tan X, Ma Z, Yan L, Ye W, Liu Z, Liang C. Radiomics nomogram outperforms size criteria in discriminating lymph node metastasis in resectable esophageal squamous cell carcinoma. Eur Radiol. 2019;29(1):392-400.

28. Asuncion Esteve-Pastor M, Miguel Rivera-Caravaca J, Roldan V, Vicente V, Valdes M, Marin F, Lip GYH. Long-term bleeding risk prediction in 'real world' patients with atrial fibrillation: comparison of the HAS-BLED and ABCbleeding risk scores. Thromb Haemost. 2017;117(10):1848-58.

29. Esteve-Pastor MA, Rivera-Caravaca JM, Roldan V, Vicente V, Valdes M, Marin F, Lip GYH. Long-term bleeding risk prediction in 'real world' patients with atrial fibrillation: comparison of the HAS-BLED and ABC-bleeding risk scores. The Murcia atrial fibrillation project. Thromb Haemost. 2017;117(10):1848-58.

30. Garcia-Fernandez A, Roldan V, Rivera-Caravaca JM, Hernandez-Romero D, Valdes M, Vicente V, Lip GY, Marin F. Does von Willebrand factor improve the predictive ability of current risk stratification scores in patients with atrial fibrillation? Sci Rep. 2017;7:41565.

31. Rodrigues G, Gonzalez-Maldonado S, Bauman G, Senan S, Lagerwaard F. A statistical comparison of prognostic index systems for brain metastases after stereotactic radiosurgery or fractionated stereotactic radiation therapy. Clin Oncol (R Coll Radiol). 2013;25(4):227-35.

32. Zhang WT, Zhang WW, He ZY, Sun JY, Zhang L, Xia Q, Wu SG. Comparison of the effects of local treatment strategies in non-metastatic Ewing sarcoma of bone. Expert Rev Anticancer Ther. 2018;18(5):501-6.

33. Zeng X, Zhang Y, Kwong JS, Zhang C, Li S, Sun F, Niu Y, Du L. The methodological quality assessment tools for preclinical and clinical studies, systematic review and meta-analysis, and clinical practice guideline: a systematic review. J Evid Based Med. 2015;8(1):2-10.
34. Duchman KR, Gao Y, Miller BJ. Prognostic factors for survival in patients with high-grade osteosarcoma using the surveillance, epidemiology, and end results (SEER) program database. Cancer Epidemiol. 2015;39(4):593-9.

\section{Publisher's Note}

Springer Nature remains neutral with regard to jurisdictional claims in published maps and institutional affiliations.
Ready to submit your research? Choose BMC and benefit from:

- fast, convenient online submission

- thorough peer review by experienced researchers in your field

- rapid publication on acceptance

- support for research data, including large and complex data types

- gold Open Access which fosters wider collaboration and increased citations

- maximum visibility for your research: over $100 \mathrm{M}$ website views per year

At BMC, research is always in progress.

Learn more biomedcentral.com/submissions 